\title{
The ARICE Framework: Augmented Reality in Computing Education
}

\author{
http://dx.doi.org/10.3991/ijet.v8i6.2809 \\ Y. Wang ${ }^{1}$, G. Vincenti ${ }^{2}$, J. Braman ${ }^{1}$, and A. Dudley ${ }^{1}$ \\ 1 Towson University, Towson, MD, USA \\ ${ }^{2}$ University of Baltimore, Baltimore, MD, USA
}

\begin{abstract}
Augmented Reality (AR) is a term for a live direct or an indirect view of a physical, real-world environment whose elements are augmented by computer-generated sensory input and output including sound, graphics or haptic input and feedback mechanisms. It has been reported that the use of AR for educational purposes provided opportunities for reflection, capabilities for multiple representations, and co-design of personalized learning experiences which contribute to promote active learning. However, most of the reported applications are for students at the elementary or high school level. There are no reported formal studies on AR application on a college/university level. Based on this lack of information, several questions arise: Are the benefits unique to the elementary and high school students? If AR is used in college classrooms, will it show similar benefits? This paper proposes an Augmented Reality in Computing Education (ARICE) framework for using an AR educational system in undergraduate computing education to improve student performance, retention and learning outcomes. A research plan on how to evaluate the framework has also been presented.
\end{abstract}

Index Terms-Augmented Reality, Computing Education, Mixed Reality Learning, ARICE.

\section{INTRODUCTION}

Emerging devices, tools, media and virtual environments offer opportunities for creating new types of learning communities for students and teachers. As mobile devices become part of everyday life, the use of these tools to support learning becomes more actively explored by educators and researchers. Augmented Reality (AR) is a term for a live direct or an indirect view of a physical, real-world environment whose elements are augmented by computer-generated sensory input and output, such as sound, graphics, or haptic input and feedback mechanisms. By superimposing information in real time within the context of learning, students can experience the world though a mixed-reality, a constructivist pedagogical approach as it involves learning by experience. Various benefits of using AR to enhance learning have been reported in the literature such as learning based on collectively seeking, sieving, and synthesizing experiences, rather than individually locating and absorbing information from some single best source; active learning based on experience that includes frequent opportunities for reflection; expression through non-linear, associational webs of representations rather than linear "stories"; and co-design of learning experiences personalized to individual needs and preferences [1].
Currently, the use of augmented reality for learning has been focused on areas of physics, geography, geometry and environmental sciences; and most of the reported applications are for students at the K-12 grade level (elementary through high school level). However, there was no formal study on the possibility of augmented reality applications at the college level. There is a serious lack of AR usage in the computing curricula at a college or university level. Using Augmented Reality (AR) applications in the area of computer science will allow for the leverage of the interactive and multifaceted benefits of the technology to enhance pedagogical techniques and motivation of students towards learning complex course materials. This paper proposes the Augmented Reality in Computing Education (ARICE) project framework that incorporates AR technology in undergraduate computing education with the goal of achieving the following objectives:

- Achieve higher learning results for students in undergraduate computing courses;

- Allow students to involve multiple senses (e.g. hearing in addition to seeing) to receive information;

- Implement "learning by doing" more effectively;

- Provide more effective learning for different target groups, especially those with low motivation and different/specific educational needs;

- Provide a positive impact on learners of different level of experiences including students with ordinary and different needs by providing a better learning experience;

- Provide "contextual learning experience" which makes it ubiquitous learning (learning anywhere, anytime).

The contents of the paper are organized as follows: First, a brief discussion on the motivation and objectives for the proposed ARICE project; Second, we present a brief literature review on AR technology in section 2 . Third, in section 3 we present AR applications in the educational field; Fourth, section 4 we present the current state of AR toolkits. And finally, in section 5 we present the details of the ARICE project; followed by our evaluation plan in section 6 and discussion on the implications of this framework and future research directions at the end.

\section{OVERVIEW OF AR}

The concept of AR has existed for nearly two decades $[2,3]$. Even though its origins can be traced even further back to the 1960 s $[4,5]$, it is only recently that this approach to human-computer interaction and immersive 
interfaces has sprung forth major interest in the general public. The change can be attributed to the lower cost of reasonably powerful technologies, now available to us in many forms, such as smart phones, tablets or laptops with integrated cameras.

The ease with which users can access devices that enable a mixed reality experience has brought to the masses the flexibility and power of augmentation. Examples of this quick change are found in the significant availability of applications for portable technologies such as the iPhone $[6,7]$ Android-based smart phones [8, 9], or even for desktop and laptop systems connected to a web camera [10].

The attention that augmented reality is receiving through the increase in commercial applications offers a significant starting point for developing applications that go beyond simple games. The portability and affordability of modern AR-enabling devices opens a wide area of research in education and other domains that was previously untapped because of limited access to the proper equipment. Augmented Reality refers to an area within the ideal gap between interacting with anything or anyone in real life and doing so in completely virtual environments. Any interaction that does not belong to either of the two extremes we just mentioned is generally referred to as mixed reality, as shown in Fig. 1 and described by Milgram and colleagues[11]. Augmented Reality in particular refers to the range of interactions that are closer to the real world, thus interactions aided through the use of computer systems that interact dynamically with the real environments in which the users operate. Fig. 2 shows a general AR system architecture which offers some insight into the inner working of this technology.

This structure should remain relatively flexible, as devices may have integrated components that perform more than one function described in Fig. 2, at any given time. For this reason we would like to also introduce a representative data flow diagram that summarizes the information in Fig. 2, abstracting it from the firm boundaries that are often found in system architectures. The data flow shown in Fig. 3, offers a key interpretation of any ARbased system, which will also serve as the technical foundation of this framework.

Researchers published a thorough survey of technologies and components that play significant roles in the implementation of AR-based solutions [5]. They address primarily output devices and methodologies, and then explore different solutions for managing the inputs. When discussing methods of delivery of the information, the visual component is the most preferred. This technology allows for flexibility and an easy blending of the real world with simulated images by having video see-through, optical see-through and projective solutions. Examples of the visual methods are given in Table I. The authors also discussed the possibility of aural interfaces, so systems can deliver augmented reality through our sense of hearing.

Depending on the type of display, we can choose different hardware solutions to output the visual interface. Generally we can utilize head-worn displays, hand-held devices, and spatial displays. Further description of these technologies is given in Table II.

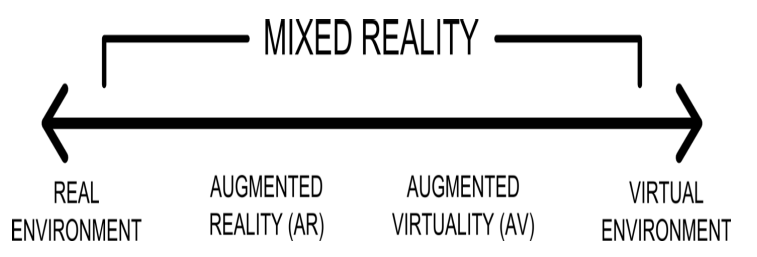

Figure 1. Reality-virtuality continuum [11]

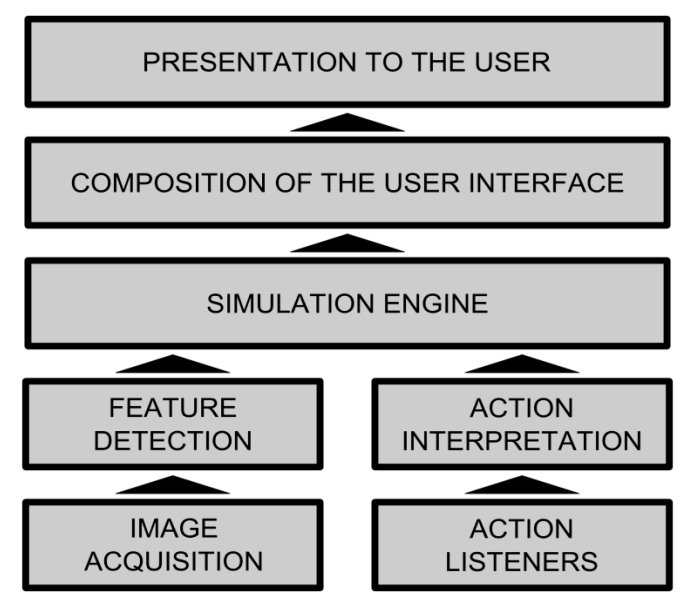

Figure 2. System architecture of a typical AR-based system

TABLE I.

EXAMPLES OF VISUAL DISPLAYS [5]

\begin{tabular}{|l|l|}
\hline \multicolumn{1}{|c|}{ Display Type } & \multicolumn{1}{c|}{ Description } \\
\hline Video see-through & $\begin{array}{l}\text { A camera captures the environment around } \\
\text { the user and augments it by adding 2- } \\
\text { dimensional and 3-dimensional structures, } \\
\text { producing an overall output to be displayed } \\
\text { on a monitor. [12] }\end{array}$ \\
\hline Optical see-through & $\begin{array}{l}\text { Transparent devices (such as glasses or } \\
\text { other similar items) are positioned between } \\
\text { the user's eyes and the environment. The } \\
\text { system then projects the virtual objects and } \\
\text { displays onto the devices, which will super- } \\
\text { impose the output to what the user can see. } \\
\text { [13] }\end{array}$ \\
\hline Projective & $\begin{array}{l}\text { The system utilizes a projector to display } \\
\text { the information directly on the environ- } \\
\text { ment. [14] }\end{array}$ \\
\hline
\end{tabular}

It is particularly important to state that even though the majority of hardware solutions reported in Table II are designed for individual use, we can build collaboration into the environment by letting users interact within the simulation [15]. Spatial displays are ideal for AR-based systems that thrive on collaboration [16]. The technologies discussed so far accommodate for the traditional outputs of AR-based systems. In addition to simple visualization and sound reproduction, research has also been done on including the sense of touch to augmented reality through haptic interfaces [17]. 


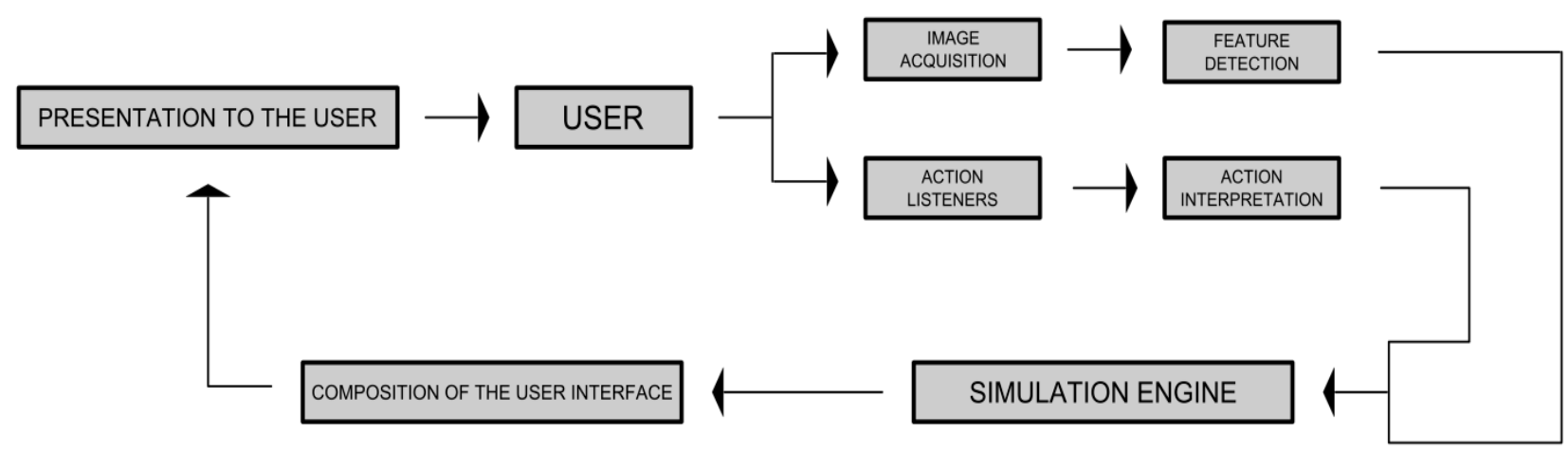

Figure 3. Data flow diagram for a typical AR-based system

The methods and technologies discussed so far are used to represent the simulation to the user, effectively taking care of the process labeled "Presentation to the user" in Fig 3. The second interface available to the user involves the collection of the inputs, and it can be composed of different processes. The idea of augmented reality involves some degree of interaction with the environment, making it necessary for the system to gather information from a camera through the "Image acquisition" process. The system then performs feature detection to identify any elements with which it is able to interact. Such elements can be objects presented by the environment and identified through feature recognition [18] or artifacts introduced by the researchers [19] in the form of 2-dimensional barcodes, called Quick Response (QR) codes [20]. In the studies previously reported, we can identify interaction mechanisms based on direct manipulation, whether the feature recognition is based on objects present in the environment and recognized by the system or through the scanning of QR codes.

The direct manipulation of the environment is not the only method of inputting information into an AR-based system. We can also utilize interfaces that directly signal to the system about how we are interacting with the simulation. The majority of video see-through technologies have the ability of projecting the images directly on touchscreens, which allow for the use of technologies such as styluses [21]. Of course, depending on the type of application, we can also resort to standard joysticks [22] or special manipulators realized ad-hoc for the system [23].

TABLE II.

EXAMPLES OF VISUAL DISPLAYS [5]

\begin{tabular}{|c|c|}
\hline Hardware Solution & Description \\
\hline Head-worn displays & $\begin{array}{c}\text { Special glasses and head-mounted monitors } \\
\text { typically utilized for video or optical see- } \\
\text { through visual displays. [13] }\end{array}$ \\
\hline Hand-held devices & $\begin{array}{c}\text { Portable computers such as smart phones or } \\
\text { tablets typically utilized for video see- } \\
\text { through display types. [12] }\end{array}$ \\
\hline Spatial displays & $\begin{array}{c}\text { Static displays that project the output on } \\
\text { surfaces, typically utilized in projective } \\
\text { display types. [14] }\end{array}$ \\
\hline
\end{tabular}

As AR-based simulations often create environments that go well beyond one's field of vision, we should also include input that are not explicitly given through motions performed by the user's arms or fingers, but instead by the position of their body. Particularly, we can collect information about the user's location through some type of positioning system such as the Department of Defense's Global Positioning System [22]. We can also utilize different sources of information to gain a more in-depth perspective of the user's location as well as orientation by adding to the GPS coordinates also information retrieved through a compass, which will help the system track what the user is seeing [24]. There have been other innovative approaches that aim at incorporating location information with audio augmentation using smart phones to assist visually impaired users [25].

\section{AR IN EDUCATION}

Mobile learning with AR can provide students unique experiences that are consistent with successful pedagogical strategies such as hands-on learning, group collaboration, concept visualization, etc. Dede [1] discussed learning styles enhanced by AR which include: fluency in multiple media; learning based on collectively seeking, sieving, and synthesizing experiences, rather than individually locating and absorbing information from some single best source; active learning based on experience that includes frequent opportunities for reflection; expression through non-linear, associational webs of representations rather than linear "stories"; and co-design of learning experiences personalized to individual needs and preferences.

AR is experiential by nature. Students are physically and perceptually involved in the experience. With the integration of a reality and simulated view, we experience the environment as if it were real. Educators and researchers have reported many attempts of using AR for learning purposes [26, 27]. It is observed that the usage of AR interfaces for computer-aided instruction to be highly engaging, especially among the students who presented behavioral and academic challenges. Most of the projects reported are introducing AR games that combine physical action with virtual interactivity. For example, Squire [28] worked on an AR game that asked students to work as environmental detectives with GPS-equipped PDA devices and walk around an outdoor site. Researchers [29] reported their efforts in building 3D models to help students understand mathematics and geometry concepts. Various impacts of AR on students' learning have been reported $[30,31]$ : 
- Achieve higher learning results

- A positive impact on learners of different age groups including students with ordinary and special needs.

- Involve multiple sensors (e.g. hearing, touching in addition to seeing) to receive information

- More effectively implement "learning by doing"

- More effective for different target groups especially those with low motivation and special educational needs

- Attract students with learning difficulties by presenting the learning process as a game

- Provide "contextual learning experience" which makes it ubiquitous learning

- $\quad$ Provide a more immersive environment by allowing learners to experience real feelings and emotions as they do in a real world through interacting with the virtual environment.

However, previous research on AR use for education was mostly done in K-12. Although educational researchers have been trying to incorporate new technologies in the classroom to enhance learning, especially in the areas of computing [32, 33], few attempts were made in utilizing AR to help students understand and remain interested in complex topics. Will we see the same kind of benefits if AR were applied for college education?

\section{AR TOOLKITS}

In the world of computing, toolkits represent a collection of routines that carry out frequently utilized features. Programmers can easily replicate complex behaviors, such as the creation of elaborate cross-platform graphical user interfaces, through the use of premade bits of code that streamline the work of developers. Toolkits are not simply an accessory that developers may choose to utilize. These libraries can be seen as a metric to gauge the advancement of a particular field, as they represent a milestone in the acceptance of a technology. Generally when one or more research groups start exploring new technologies, they are forced to create their own tools to accomplish their goal. Once the scientific community receives and approves the results, the need to facilitate the recreation of the tools utilized in the original research increases in order to reduce the time necessary to reach the point where the team is focusing on research rather than reimplementation.

TABLE III.

ARTOOLKIT API [39]

\begin{tabular}{|l|l|}
\hline \multicolumn{1}{|c|}{ Data Structure } & \multicolumn{1}{c|}{ Description } \\
\hline ARMarkerInfo & $\begin{array}{l}\text { Main structure for detected mark- } \\
\text { er }\end{array}$ \\
\hline ARMarkerInfo2 & $\begin{array}{l}\text { Internal structure use for marker } \\
\text { detection }\end{array}$ \\
\hline ARMat & Matrix structure \\
\hline ARMultiEachMarkerInfoT & Multi-marker structure \\
\hline ARMultiMarkerInfoT & Global multi-marker structure \\
\hline ARParam & Camera intrinsic parameters \\
\hline arPrevInfo & $\begin{array}{l}\text { Structure for temporal continuity } \\
\text { of tracking }\end{array}$ \\
\hline ARVec & Vector structure \\
\hline
\end{tabular}

In the case of Augmented Reality we can easily identify several toolkits that are available to developers: ARToolKit [34], SLARToolkit [35], NyARToolkit [36], FLARToolKit [37] and DART [38]. If we look closely to all these toolkits, we can identify a common root in ARToolKit, which serves as the base technology to the other libraries. This phenomenon can be traced back to the fact that augmented reality connects directly the hardware with impressive graphical user interfaces, traversing the entire stack of technologies that create computers. The need for high performance coupled with the need of adaptability to each system's hardware as well as software resources requires a high degree of tailoring for a particular toolkit to different technologies. For example, the original ARToolKit is written in $\mathrm{C}++$ and is accessible by many platforms. Projects such as SLARToolkit and FLARToolKit utilize not only the original ARToolKit but couple its power with other development kits, such as SilverLight (SLARToolkit) or Flash (FLARToolKit). Other toolkits are tailored instead to the needs of a niche, such as DART (aimed at designers).

Even though the presence of a toolkit may reveal the maturity of a particular technology's acceptance in society, we have to realize that different projects have different needs. As there are no two projects that are identical in platforms, goals, and algorithms it is difficult for programmers to realize a particular set of data structures that can satisfy any need. We can validate this fact by acknowledging the relatively small set of features that is offered by ARToolKit, reported in Table III.

After reviewing the API, we can notice that the bulk of the operations relates to the detection of markers, utilized to signal to the program where in the camera's view the interactive content should exist, and their tracking through the operations of the system. This component is extremely important, and relatively universal through AR-based applications that thrive on markers. If we were to utilize a GPS and compass, this particular technology would not help us.

Perhaps the most important aspects that we should notice involve the interfaces with other systems, namely the acquisition system and the AR engine. ARToolKit does not offer any data structures or mechanisms that power the actual display of the information; as such functionality needs to be offered by an API that is specific to the system on which the user is operating. This toolkit is able to integrate a basic tool for interacting with a camera, which is generally used to acquire the information that will then drive the user's experience.

\section{ARICE FRAMEWORK.}

College student retention has always been an issue in higher education and has recently come back into the national spotlight due to the surprising results of an international survey ranking college degree holders. A recent study by the Consortium for Student Retention Data Exchange at The National Symposium on Student Retention [40] revealed that the effectiveness of the degree program was the third highest reason for dropping out -12.3 percent and 13.7 percent, for men and women respectively. Similar to national trends, at our university (a metropolitan university in mid-Atlantic region), six-year graduation rate for 2004 cohort is at $68.2 \%$ (http://www.towson.edu/ ir/cdsindex.asp). President Obama has issued a call for 
more college graduates by 2020 . For the computing field, the picture is not different from the rest of the educational areas. How can we attract high school students to this field, especially underrepresented groups? How can we make our program more effective so that we can retain and better teach students once they are in school? Based on the benefits of AR in education reported from the literature, we believe appropriate utilization of AR technology will be able to help. Therefore, we propose the design, delivery and testing of an AR based learning environment for computing education.

Lower level computing courses are often the classes that start introducing some complex concepts which have quite a few students turned away because of the difficulty of absorbing the materials. Making lower level computing courses more effective in the delivery of content may present a higher opportunity to retain students and to motivate students to learn more in computing field. More importantly, some of the materials can be used as recruitment tools for high school graduates by creating an interest in technology and computing, thus increasing the number of potential students who may pursue a degree in a computer related field. Therefore, lower level computing courses are good candidate as testing bed for evaluating the framework we proposed here.

The ARICE framework will involve the design of interactive games that help students learn computing concepts including:

- An AR based learning system that provides a platform to present learning materials in various formats;

- Learning materials that best utilize AR features to provide better learning experience to undergraduate students and enhance their learning; and

- A Learning game that provides interaction for students to learn and help evaluate their learning outcome.

\section{A. AR Based Learning System}

The ARICE framework will involve the design and implementation of an AR based learning system with the architecture discussed in Fig. 2. With the availability of mobile devices (such as android based phones or tablets), hand-held devices will be used as the hardware solution for this system. Students will be able to use the hand-held devices to access course materials and work on interactive exercises as discussed in detail in section B.

\section{B. Design of the Learning Materials and Exercises}

In order to provide effective ways to deliver learning materials with an AR based learning system, considerations must be given so that it best utilizes AR features while keeping students engaged. To learn concepts in the computing field, it is essential that students can learn them by doing it. Therefore, the learning exercise must provide "hands-on" capabilities. In other words, students should be able to do it and see the result right away so that they can interpret the impact of their actions. Considering different learning style and different needs from students, it is imperative that learning materials are presented in different ways (involving video, audio, text, etc.)

In addition, the learning exercises will have different levels of learning built in for each concept covered. As illustrated in Fig. 4, Students will start from a simple exer- cise that tests their understanding on a specific concept. Once the system interprets that the student is able to do the exercise correctly, a more complicated challenge will be presented. The higher level of challenge a student encounters, the higher the award (scores) for finishing it correctly will be. As the semester goes by, students will be able to go through a set of higher level challenges. Ideally, they will be able to finish the synthesis of multiple concepts learned throughout the semester. Since the AR system is also available through their mobile hand-held devices, it is possible that students can learn the materials whenever and wherever they want. They don't have to be confined in a classroom to learn the materials.

For example, when discussing introductory programming concepts, learning modules will focus on computer programming fundamentals, such as IF-Statements, While-Loops, For- Loops and Arrays. Other computing fundamentals, such as stacks, queues, trees, graphs and classes, will also be included. Fig. 5 illustrates an example of four main modules and their order relations. Once a student has successfully progressed through one module, they can proceed to the next concept. Students are allowed to progress backwards to reinforce material if needed, but not allowed to move forward until they have successfully demonstrated their mastery of a particular skill through built in assessments (hands on exercises).

Each main content module will consist of the following components: Text, Video, a 2D Interactive Demo, an AR Interactive Demo and an Assessment.

Text- Each concept will be explained via text examples as in traditional learning. Examples and information will be presented along with important information. This component can be achieved by using a slideshow presentation approach.

Video- Each concept will have a video tutorial complete with animation and sound. The video component can

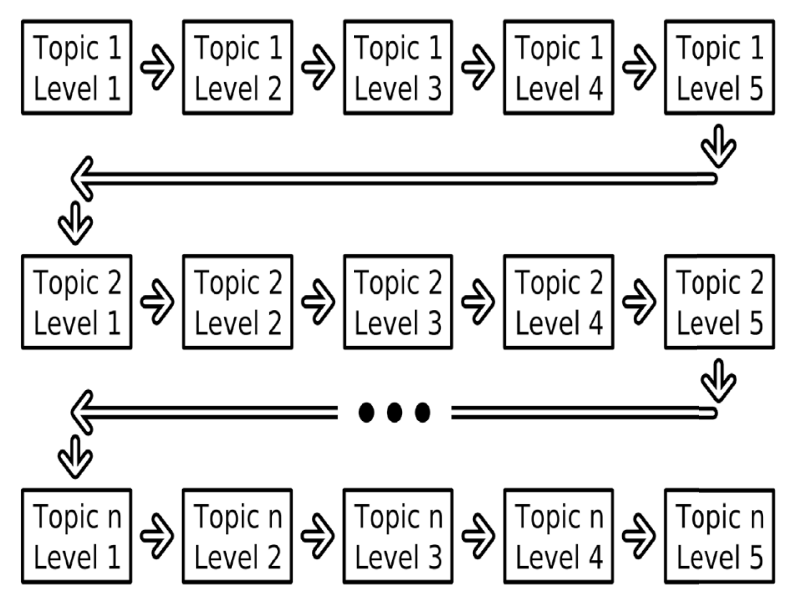

Figure 4. Learning exercises structure illustration

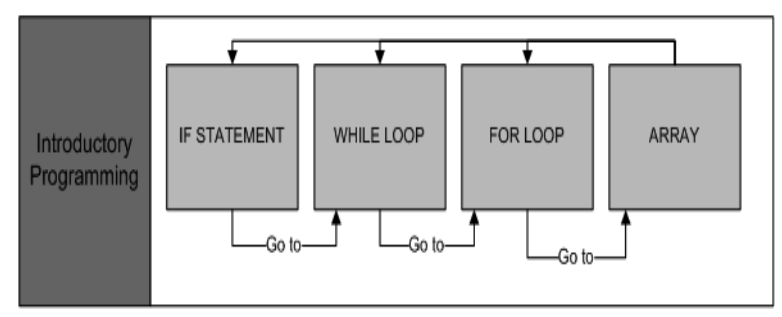

Figure 5. Asset overview for introductory programming concepts 
be used to demonstrate concepts. For example, to illustrate the idea of a While-Loop, users can be presented with various examples and animations of concepts from the real world. There could be an image of someone scrubbing clothes, and each time it is washed, we can check the condition: While the clothes are not clean, continue washing the clothes.

2D Interactive Demo - Interactive learning objects can be used to demonstrate a concept. With this component, students have the ability to directly interact with an adaptable example. By changing the parameters, the problem and solution will change. Students can use this approach to see how the concept behaves in different scenarios.

Interactive AR Demo - Similar in idea to the 2D interactive Demo, the AR Demos will be designed to be manipulated by the students for a simulated "hands-on" approach. Students can scan the bar-code to be read by a mobile or tablet device, which will display the AR component. For example, if students are leaning about Arrays, Trees or even Tree rotations, they can manipulate a replica of the problem directly to see the results immediately.

Assessment - To advance to the next level of learning, students must pass a hands-on exercise as a "quiz". Scores will be granted once they do the exercise correctly. Once they earn scores to a preset threshold, they will be allowed to go to the next level. Students will be allowed multiple attempts for the exercises in order to help them learn. This kind of assessments will be built in at the end of each learning module.

As students will have access to these modules in the classroom via table devices or by using their own mobile phone or device, these components will be available as the students need them. Modules will be designed in a gamelike fashion in order to encourage students to practice and learn. To access the materials, the device can scan a Quick Response code (QR code) that will be associated with problems and concepts taught in class (See Fig. 6). The system can also display an interactive AR component of the problems that students can manipulate.

\section{Course Delivery as Pilot Study}

In order to verify the validity of the framework proposed, a pilot study to investigate how AR can be infused into introductory computing courses as a teaching tool will be conducted. One main limitation of our current framework is the lack of supporting data. Student feedback on the usage and effectiveness of this tool will be collected as part of effort to evaluate this framework. The following courses listed in Table IV are some possible courses that can be used for pilot study.

TABLE I.

SELECTED COURSES AND OF DESCRIPTIONS

\begin{tabular}{|l|l|}
\hline \multicolumn{1}{|c|}{ Course } & \multicolumn{1}{c|}{ Description } \\
\hline $\begin{array}{l}\text { General Comput- } \\
\text { er Science }\end{array}$ & $\begin{array}{l}\text { Computer systems overview, algorithm devel- } \\
\text { opment, data presentation, software design and } \\
\text { testing methodologies and brief overview of } \\
\text { advanced topics. }\end{array}$ \\
\hline $\begin{array}{l}\text { Computer Sci- } \\
\text { ence 1 }\end{array}$ & $\begin{array}{l}\text { Introduction to structured problem solving, } \\
\text { algorithm development and computer pro- } \\
\text { gramming with a modern high-level structured } \\
\text { programming language. Three lecture hours and } \\
\text { two laboratory hours. }\end{array}$ \\
\hline
\end{tabular}

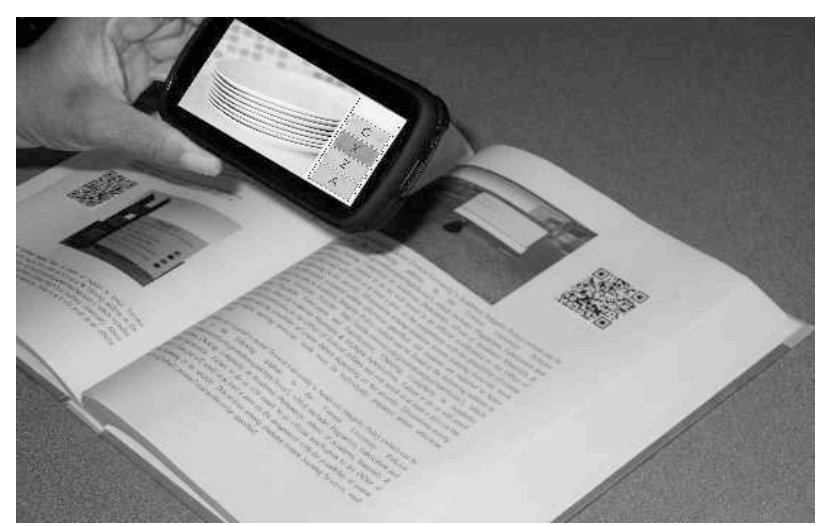

Figure 6. Concept demonstration using a cell phone

After the implementation of the AR learning system and the design of all the course materials, a usability study will be conducted before the formal delivery of the course to make sure that the system and the materials can be accessed easily by the students with their hand-held devices. After applying necessary improvement based on the result from the usability study, one section of each course identified above will incorporate the new AR learning materials in the course delivery in addition to traditional classroom discussion. Student usage data will be recorded to check whether students really take advantage of the new materials. More detailed discussion on how to evaluate the effect of AR based learning can be found in section 6 .

\section{EVALUATION PLAN}

Once the usability test of the AR learning system and learning game is completed, a formal evaluation of this framework will be conducted to investigate the effects of such a framework towards students learning experiences. This evaluation will address questions such as: How does the use of the AR game affect students' learning? Do the students experience the same set of benefits identified in the literature? In addition, the evaluation will try to investigate whether the anticipated outcome has been reached:

- Using the interaction features of the Augmented Reality interactive game in a computing course will help students better understand the learning materials. Therefore, it is surmised that students should achieve higher learning results.

- Using the highly engagement features of the Augmented Reality interactive game in a computing course will motivate students to learn more. Therefore, it will help retain students, students' having learning disabilities, and introduce different learning styles.

To evaluate the above outcomes, both qualitative and quantitative approaches will be adopted. Interviews with the instructor as well as at least two randomly selected students from each class will be conducted to collect their overall experience on the augmented reality interactive game for the class they attended. In addition, a system log will be utilized to indicate the time students spent playing the games and their progress. Questionnaires containing 30 questions will also be distributed to students. The questionnaire will include 10 questions on technology background and learning styles, 10 on affective checklist, 10 reflective questions. The 30 question survey with at least 


\section{SPECIAL FOCUS PAPER \\ THE ARICE FRAMEWORK: AUGMENTED REALITY IN COMPUTING EDUCATION}

75 participants for each group will examine the interaction of AR success with self-identified learning styles and or needs. In addition, video-taped focus groups on student's AR learning and impact will be conducted.

Although pass/fail rate of the class cannot be utilized as a powerful indicator to illustrate the improvement in learning outcome comparing to previous classes that did not involve such games, it can still serve as one of the indicators of students' learning outcome. Another learning outcome indicator can be reflected by the quality of the student final projects. These reports will be evaluated against previous student projects to see whether there is any improvement.

In addition to the above set of data, to investigate whether the use of AR helps retain students can be done by the following common activities conducted for each semester from each course:

- Number of students enrolled at end of two weeks

- Number of students withdrawn from class

- Number of students completing the course, but dropping major

- Collect the same data from matched course with no AR treatment

\section{CONCLUSION}

While educating students on complex topics in the undergraduate computing curriculum is a top priority for educators and for the future success of the computer field, it can be extremely difficult and often challenging. Although many innovative educational technologies exist and are in use, augmented reality has been used in limited contexts. In this paper we have discussed several aspects of the ARICE project framework. This framework is specifically aimed at enhancing undergraduate computing education by providing multiple modes of content delivery through a virtual augmented reality. We discussed key elements of AR technology such as presenting how it can be used in an educational context and current toolkits followed by our AR Framework (ARICE). We have also presented an evaluation plan for the success of this approach once we complete our pilot study as a formal test of the framework. Currently, the development of a prototype of the AR based learning system is underway and will be tested with lower level computing courses as previously identified.

With the success from the previous adoption of AR in $\mathrm{K}-12$ system, the authors see AR technology as a means to further enhance learning. Using AR technology is a practical way to learn by; which is a method of self-paced education. Once the ARICE framework is fully implemented, it has the potential to be a powerful learning tool. Students could use this learning module as an extension to classroom learning as a way to practice concepts on their own. Through the framework, students can manipulate AR content to understand complex topics. Moreover, this framework could be used in many other areas besides computing education. An AR system can accommodate many types of materials such as: various visual displays and manipulations and text intensive and graphical intensive domains could benefit from the applications of this technology. For example, domains ranging from mathematics (formula heavy / text) to engineering, where 3D models may be more common and necessary may significantly benefit from the application of this framework.

The authors are currently seeking innovative approaches to test this framework in their current courses. The next main goal in the project is to use the framework in the previously identified computing courses on some topics in order to collect feedback on the system, its usability and its effectiveness. In the collection of data from our courses, once the framework is validated, the authors intended to extend this framework to other computing courses. Another goal through ARICE is to provide educators a foundation and a tool to enhance their curriculum for the advancement of computing education.

\section{REFERENCES}

[1] C. Dede, "Planning for Neomillennial Learning Styles". Educause Quarterly, 28(1): pp. 7-12. 2005.

[2] P. Wellner, W. Mackay, and R. Gold, "Computer Augmented Environments: Back to the Real World". Special Issue of the Communications of the ACM, 36(7), pp. 24-26. 1993.

[3] W.E. Mackay, "Augmented Reality: Linking real and virtual worlds. A New paradigm for interacting with computers". Proceedings of the working conference on advanced visual interfaces, pp. 13-21. 1998.

[4] H. Tamura, "Steady steps and giant leap toward practical mixed reality systems and applications". Proceedings of the International Status Conference on Virtual and Augmented Reality. 2002.

[5] D.W.F. van Krevelen and R. Poelman, "A Survey of Augmented Reality Technologies, Applications and Limitations". The International Journal of Virtual Reality, 9(2), pp. 1-20. 2010.

[6] Golfscape. Homepage. Retrieved from http://golfscapeapp.com/

[7] Panoramascope. Retrieved from http://panoramascope.com/

[8] Layar. Homepage. Retrieved from http://www.layar.com/

[9] Wikitude Homepage. Retrieved from http://www.wikitude.org/en/

[10] Hallmark. Webcam Greetings Cards. Retrieved from http://www.hallmark.com/online/webcam-greetings.aspx

[11] P. Milgram, H. Takemura, A. Utsumi, and F. Kishino. "Augmented Reality: A Class of displays on the Reality-Virtuality Continuum". Telemanipulator and Telepresence Technologies, 2351, pp. 282-292. 1994.

[12] T. Miyashita, P. Meier, T. Tachikawa, S. Orlic, T. Eble, V. Scholz, A. Gapel, and O. Gerl, "An Augmented Reality Museum Guide". 2008. IEEE International Symposium on Mixed and Augmented Reality 2008, pp. 103-106.

[13] O. Oda, L.J. Lister, S. White, and S. Feiner, "Developing an Augmented Reality Racing Game". Proceedings of the 2nd International conference on Intelligent Technologies for Interactive Entertainment, pp. 1-8. 2008. http://dx.doi.org/10.4108/ICST.INTE TAIN2008.2472

[14] M.R. Marner, B.H. Thomas and C. Sandor. "Physical-virtual tools for Spatial Augmented Reality User Interfaces". Proceedings of the 2009 8th IEEE International Symposium on Mixed and Augmented Reality, pp. 205-206. 2009. http://dx.doi.org/10.1109/ ISMAR.2009.5336458

[15] A.C.M. Tavares, S.M.M Fernandes, and M.L.P. de Menezes, "NHE: collaborative virtual environment with augmented reality on web". 2010 International Conference on Cyberworlds, pp. 438444.

[16] A.W. Ismail and M.S. Sunar, "Survey on Collaborative AR for Multi-user Urban Studies and Planning". ICIMT '09 Proceedings of the 2009 International Conference on Information and Multimedia Technology. pp. 19-23. 2009.

[17] S. Jeon, and S. Choi, "Haptic Augmented Reality: Taxonomy and an Example of Stiffness Modulation". 2009. Presence, 18(5), pp. 387-408. http://dx.doi.org/10.1162/pres.18.5.387

[18] S.J. Henderson, and S. Feiner, "Evaluating the benefits of augmented reality for task localization in maintenance of an armored personnel carrier turret", IEEE International Symposium on Mixed and Augmented Reality 2009 Science and Technology Proceedings, pp. 135-144. 2009. 


\section{SPECIAL FOCUS PAPER \\ THE ARICE FRAMEWORK: AUGMENTED REALITY IN COMPUTING EDUCATION}

[19] X. Wang, and R. Chen, “An Experimental Study on Collaborative Effectiveness of Augmented Reality Potentials in Urban Design". CoDesign, 5(4), pp. 229-244. 2009. http://dx.doi.org/10.1080/157 10880903320020

[20] D. Ley, "Ubiquitous Computing”. In Emerging Technologies for Learning. (Vol. 2, pp. 64-79). Coventry, UK: British Educational Communications and Technology Agency (Becta). 2007.

[21] C. Aguiló, J. Lorés, and E. Junyent, "Enhanced cultural heritage environments by augmented reality systems". Proceedings of the Seventh International Conference on Virtual Systems and Multimedia. 2001.

[22] A. Hammad, H. Wang, and S.P. Mudur, "Distributed augmented reality for visualizing collaborative construction tasks". Journal of Computing in Civil Engineering, 23(4), pp. 418-427. 2009. http://dx.doi.org/10.1061/(ASCE)0887-3801(2009)23:6(418)

[23] H. Iseki, Y. Muragaki, K. Naemura, M. Hayashi, T. Hori, and K. Takakura, "Clinical application of augmented reality in neurosurgical field". Proceedings of the Computer Graphics International, pp. 1-6. 2003.

[24] B. Thomas, B. Close, J. Donoghue, J. Squires, P. De Bondi, and W. Piekarski, "First Person Indoor/Outdoor Augmented Reality Application: ARQuake". Personal and Ubiquitous Computing, 6, pp. 75-86. 2002. http://dx.doi.org/10.1007/s007790200007

[25] J. Blum, M. Bouchard, J.R. Cooperstock, "What's around me? Spatialized audio augmented reality for blind users with a smartphone". In Mobile and Ubiquitous Systems (Mobiquitous). 2011.

[26] V. Lamanauskas, and R. Bilbokaite, "Pedagogical Evaluation of Prototype 3 of the AR Learning Platform Based on the Results Achieved During the Third ARISE Summer School". Problems of Education in the $21^{\text {st }}$ Century, Vol. 11, pp. 86-103. 2009.

[27] M. Dunleavy, C. Dede, and R. Mitchell, "Affordances and Limitations of Immersive Participatory Augmented Reality Simulations for Teaching and Learning". Journal of Sciences Education and Technology, 18: pp. 7-22. 2009. http://dx.doi.org/10.1007/s10956008-9119-1

[28] K. Squire. "From Information to Experience: Place-Based Augmented Reality Games as a Model for Learning in a Globally Networked Society". Teachers College Record. 112(10): pp. 25652602. 2010

[29] H. Kaufmann, and D. Schmalstieg, "Mathematics and Geometry Education with Collaborative Augmented Reality". Computers and Graphics, Vol. 27, pp. 339-345. 2003. http://dx.doi.org/10.1016/S0097-8493(03)00028-1

[30] M. Vilkoniene, M. Lamanauskas, and R. Vilkonis, "Pedagogical evaluation of the teaching/learning platform based on augmented reality technology: The opinion of science teachers". Information \& Communication Technology in Natural Science Education, pp. 181-211. 2007.
[31] M. Vilkoniene, "Influence of augmented reality technology upon pupil's knowledge about human digestive system: The results of the experiment". US-China Education Review, 6(1): pp. 36-43. 2009.

[32] G. Vincenti, and J. Braman, Teaching through Multi-User Virtual Environments: Applying Dynamic Elements to the Modern Classroom. Information Science Reference. 2010. http://dx.doi.org/10.4018/978-1-61692-822-3

[33] M. DiBlasio, G. Vincenti, and J. Braman, "Designing SCORM Compliant Courses for Introductory Programming Students". Proceedings of the $3^{\text {rd }}$ Symposium on Academic Globalization. Orlando, Florida, USA. 2010.

[34] ARToolKit Retrieved: http://www.hitl.washington.edu/artoolkit/

[35] SLARToolkit. Retrieved from http://slartoolkit.codeplex.com/

[36] NyARToolkit Homepage. Retrieved from: http://nyatla.jp/nyartoolkit/wiki/index.php?FrontPage.en

[37] FLARToolKit. Homepage. Retrieved from: http://www.libspark.ork/wiki/saqoosha/flartoolkit/en

[38] DART Homepage. Retrieved from http://www.cc.gatech.edu/dart/

[39] ARToolKit API http:// artoolkit.sourceforge.net/apidoc/annotated. html

[40] D. De Vise, "Non-traditional students key to college completion goal". 2011. Retrieved from: http://www.insidetrack.com/news/ view/guest-post-non-traditional-students-key-to-college- completion-goal/

\section{AUTHORS}

Y. Wang is with the Department of Computer and Information Sciences of Towson University, Towson, MD 21252 USA. (ywang@towson.edu).

G. Vincenti is with the Division of Science, Information Arts and Technologies, University of Baltimore, Baltimore, MD 21201 USA. (gvincenti@ubalt.edu).

J. Braman is with the Department of Computer and Information Sciences of Towson University, Towson, MD 21252 USA. (jbraman@towson.edu).

A. Dudley is with the Department of Computer and Information Sciences of Towson University, Towson, MD 21252 USA. (adudley@towson.edu).

This article is an extended and modified version of a paper presented at the 2011 Interdisciplinary Conference of AHLiST, held May 20-22, 2011, at College of Technology, University of Houston, TX, USA. Submitted 06 September 2011. Published as re-submitted by the authors 08 November 2013. 